\title{
La oportunidad surgida de la necesidad: Cambio metodológico en la asignatura de Construcción 1 en tiempos de pandemia.
}

\section{An opportunity from necessity: Methodological change in the Building Construction I Course in pandemic times.}

Victoria PATRICIA López CABEza

https://orcid.org/0000-0002-5257-1281

Universidad de Sevilla.

Departamento de Construcciones Arquitectónicas I.

vlopez7@us.es

DOI: http://dx.doi.org/10.12795/9788447231003.039

Pp.: 818-840 


\section{Contexto de aplicación del Ciclo de Mejora en el Aula.}

Este Ciclo de Mejora en el Aula (CIMA) se realiza en el contexto de la asignatura de Construcción I del Grado en Arquitectura, de primer curso, en el primer cuatrimestre. Se trata, por tanto, de una de las primeras asignaturas en las que los alumnos tienen contacto con la arquitectura y la vida universitaria. El grupo es de un tamaño de 29 alumnos.

La asignatura de Construcción I se centra en la introducción al diseño de los sistemas constructivos y al acondicionamiento de edificios aplicados a la tipología de vivienda unifamiliar. Dentro del bloque de acondicionamiento se tratan el acondicionamiento lumínico, térmico y acústico, y dentro del bloque de sistemas constructivos se tratan envolvente, particiones, estructura, cimentación y elementos de comunicación vertical.

La evaluación es continua. Habitualmente, las clases se estructuran con una parte en la que se presenta la teoría, de carácter expositivo por parte del profesor, y un tiempo, si sobra, que se dedica a la realización de las prácticas que se les asignan a los alumnos en cada tema, de realización individual. El modelo se representa en la Figura 1.

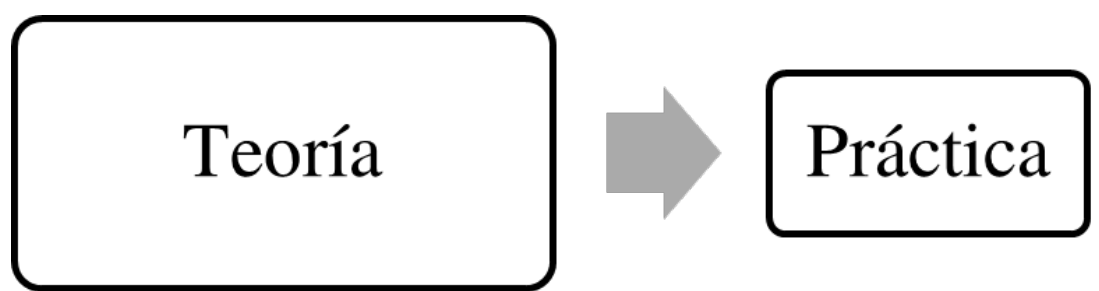

Figura 1. Modelo metodológico habitual de la asignatura de Construcción 1 del Grado en Fundamentos de la Arquitectura

Ciclos de Mejora en el Aula (2020). Experiencias de Innovación Docente de la US Esta obra se distribuye con la licencia Creative Commons 


\section{Diseño del CIMA}

En este apartado se presenta el diseño de ciclo de mejora realizado siguiendo la metodología presentada por Porlán (2017). En primer lugar, se muestra el trabajo realizado con los contenidos para seleccionarlos y jerarquizarlos, posteriormente se diseña la secuencia de actividades y finalmente se muestran las herramientas de evaluación que se van a utilizar.

\section{Mapa de contenidos}

Los contenidos de la asignatura de Construcción son extensos y estructurantes en el conjunto global del grado, pues sirven de introducción a múltiples asignaturas de otros cursos, no solo Construcción 2, sino también Instalaciones o Estructuras. Es por esto por lo que es necesario mostrarles a los alumnos la vinculación de cada contenido con el resto de su aprendizaje tanto dentro de la asignatura como en el resto del grado, para lo cual el mapa de contenidos es una herramienta imprescindible. Además, al ser una asignatura teórico-práctica, la diferenciación entre contenidos procedimentales y conceptos permite una mejor estructuración de las actividades a diseñar. Por último, la especificación en el mapa de contenidos de los contenidos actitudinales permite que no se dejen de lado, como habitualmente puede llegar a pasar en asignaturas con mucho temario con las cuestiones más transversales.

El presente CIMA se va a aplicar en el bloque de contenidos de sistemas del edificio, en concreto al tema de la envolvente. En la figura 2 se muestra el mapa de contenidos elaborado, que se estructura partiendo de la pregunta global del bloque, que es “¿Cuáles son los diferentes sistemas del edificio?". De su respuesta surge el primer

Ciclos de Mejora en el Aula (2020). Experiencias de Innovación Docente de la US Esta obra se distribuye con la licencia Creative Commons 
concepto estructurador: La envolvente como uno de los sistemas que componen el edificio. A continuación, se responde a la pregunta de "¿Qué elementos componen la envolvente del edificio?" y surgirán los dos nuevos conceptos estructurantes: Los muros y las cubiertas. De ellos surgen los tipos y los elementos que los componen. La tercera pregunta que se trata de responder en el mapa conceptual es “¿Pará qué se construye la envolvente del edificio?". De esta pregunta parten procedimientos intelectuales de relacionar los elementos de la envolvente con los diferentes materiales y funciones que deben cumplir, relacionados a su vez con la normativa (Ministerio de Fomento, 2019) y el valor de la sostenibilidad. Por último, se plantea la pregunta de “Cómo comunicamos nuestro diseño para ser construido?". Respondiendo a esta pregunta los estudiantes aprenderán los procedimientos intelectuales de saber representar sus diseños constructivos y las habilidades para hacerlo correctamente.

Ciclos de Mejora en el Aula (2020). Experiencias de Innovación Docente de la US Esta obra se distribuye con la licencia Creative Commons 


\section{VICTORIA PATRICIA LÓPEZ CABEZA}

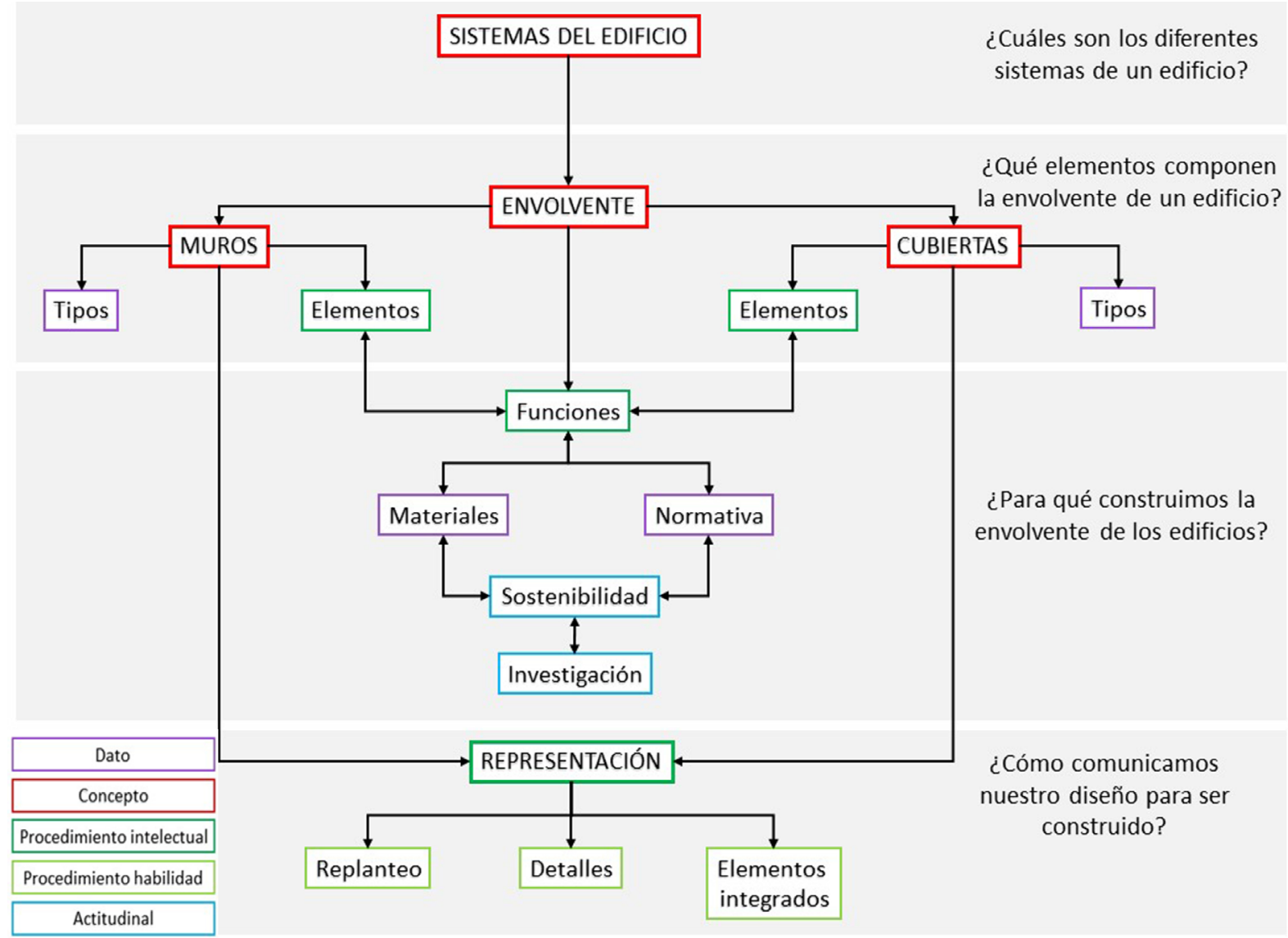

Figura 2. Mapa de contenidos elaborado para el bloque de Envolvente de Construcción 1.

Ciclos de Mejora en el Aula (2020). Experiencias de Innovación Docente de la US cc (i) $\Theta$ Esta obra se distribuye con la licencia Creative Commons 


\section{Modelo metodológico y secuencia de actividades}

El modelo metodológico posible se plantea con los objetivos de hacer al estudiante más partícipe de su aprendizaje, favorecer el trabajo de los estudiantes en clase y el aprendizaje colaborativo, que son algunos de los principios en los que se basa la metodología de los mejores profesores universitarios (Bain, 2007). Esto se traduce en una serie de actividades que fomentan esta mayor implicación por parte de los alumnos. El esquema metodológico posible se muestra en la Figura 3.

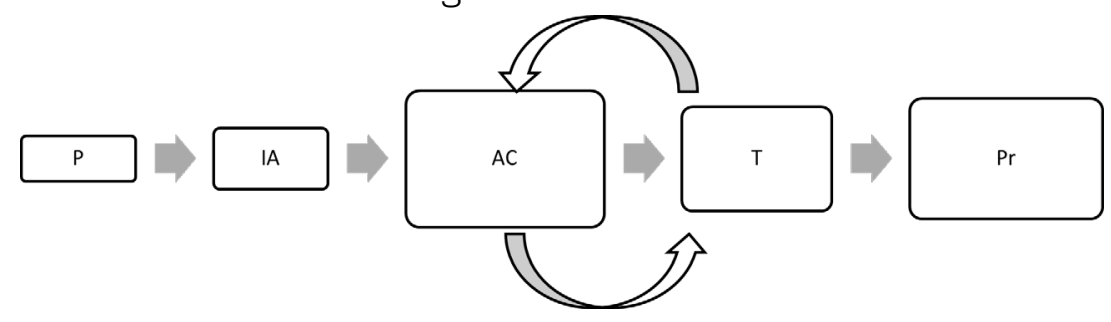

Figura 3. Modelo metodológico posible propuesto. $P=$ Problema. $I A=I$ deas de los alumnos. $T$ = Teoría. $A C=$ Actividad de Contraste. Pr=Práctica.

A continuación, en las tablas 1 a 4, se desarrolla el esquema en las diferentes actividades de cada una de las sesiones. Se trata de un total de cuatro sesiones de dos horas cada una:

Tabla 1. Secuencia de actividades de la primera sesión de aplicación del CIMA.

\begin{tabular}{|l|l|c|}
\hline \multicolumn{3}{|c|}{ SESIÓN 1 (120') } \\
\hline ACTIVIDAD & DESCRIPCIÓN TIEMPO \\
\hline Problema & $\begin{array}{l}\text { La clase se inicia con la presentación del } \\
\text { tema y la pregunta que va a estructurar todo } \\
\text { el contenido del bloque: “Cuáles son los } \\
\text { diferentes sistemas del edificio?". }\end{array}$ & 10' \\
\hline $\begin{array}{l}\text { Cuestionario } \\
\text { inicial }\end{array}$ & $\begin{array}{l}\text { Los estudiantes responden a un cuestionario } \\
\text { inicial a través de la plataforma Google Forms. }\end{array}$ & 15' \\
\hline
\end{tabular}

Ciclos de Mejora en el Aula (2020). Experiencias de Innovación Docente de la US Esta obra se distribuye con la licencia Creative Commons 


\begin{tabular}{|l|l|l|}
\hline $\begin{array}{l}\text { Actividad de } \\
\text { contraste }\end{array}$ & $\begin{array}{l}\text { Loa alumnos responden a la pregunta “Pará } \\
\text { qué se construye la envolvente del edificio?" } \\
\text { Actividad que se realiza en grupo con la ayuda } \\
\text { de la aplicación online “mentimeter" para la } \\
\text { elaboración en grupo de nubes de palabras. } \\
\text { Se comentan los resultados para explicar esa } \\
\text { parte de la teoría. }\end{array}$ & 10' \\
\hline Teoría & $\begin{array}{l}\text { El resto de los contenidos se exponen de forma } \\
\text { más tradicional pero siempre tratando de hacer } \\
\text { participar a los alumnos para que respondan } \\
\text { ellos primero a las preguntas planteadas. } \\
\text { Se diseña una secuencia de preguntas que } \\
\text { se construyen unas sobre otras e incluyen } \\
\text { experiencias familiares de manera que vayan } \\
\text { guiando el razonamiento del estudiante hacia } \\
\text { la solución (Finkel, 2008). }\end{array}$ & 30' \\
\hline $\begin{array}{l}\text { Actividad de } \\
\text { contraste }\end{array}$ & $\begin{array}{l}\text { La primera actividad de contraste para que } \\
\text { aprendan el contenido procedimental de la } \\
\text { unidad se trata de un análisis guiado de un } \\
\text { plano de replanteo de muros. Se realiza en gran } \\
\text { grupo, lanzando las preguntas y esperando a } \\
\text { que los alumnos las respondan observando un } \\
\text { ejemplo. Para profundizar en los contenidos } \\
\text { más prácticos, se realizan dos actividades de } \\
\text { contraste. Por un lado, un análisis guiado de } \\
\text { un plano de replanteo de muros. Por otro, una } \\
\text { práctica en grupo de relación de imágenes con } \\
\text { detalles constructivos. }\end{array}$ & $\begin{array}{l}\text { Para el segundo tipo de contenido } \\
\text { procedimental, la realización de detalles, se } \\
\text { plantea una segunda actividad de contraste de } \\
\text { relación de imágenes con detalles constructivos } \\
\text { e identificación de capas. La realizan en } \\
\text { pequeños grupos. Se corrige cuando uno de } \\
\text { ellos termina, promoviendo cierta competición } \\
\text { dándole puntos positivos por rapidez. }\end{array}$ \\
\hline $\begin{array}{l}\text { Se realiza una conclusión de las principales } \\
\text { ideas del día y se presenta la práctica individual } \\
\text { para que la realicen para el próximo día de } \\
\text { clase. }\end{array}$
\end{tabular}$\quad$ 20'

Ciclos de Mejora en el Aula (2020). Experiencias de Innovación Docente de la US Esta obra se distribuye con la licencia Creative Commons 
Tabla 2. Secuencia de actividades de la segunda sesión de aplicación del CIMA.

\begin{tabular}{|c|c|c|}
\hline \multicolumn{3}{|c|}{ SESIÓN $2\left(120^{\prime}\right)$} \\
\hline ACTIVIDAD & DESCRIPCIÓN & TIEMPO \\
\hline $\begin{array}{l}\text { Actividad de } \\
\text { contraste }\end{array}$ & $\begin{array}{l}\text { Corrección en pizarra de la actividad individual } \\
\text { enviada en la sesión anterior. }\end{array}$ & $20^{\prime}$ \\
\hline Teoría & $\begin{array}{l}\text { Los contenidos básicos de la teoría de cubiertas } \\
\text { planas se darán de forma expositiva, intercalados } \\
\text { continuamente con preguntas para que los } \\
\text { estudiantes participen. }\end{array}$ & $35^{\prime}$ \\
\hline $\begin{array}{l}\text { Actividad de } \\
\text { contraste }\end{array}$ & $\begin{array}{l}\text { Actividad en grupo de relación de capas y } \\
\text { funciones de las cubiertas planas sobre un detalle } \\
\text { tipo. Uso de Blackboard Collaborate en grupos. } \\
\text { Corrección en grupo, explicando resultados. }\end{array}$ & $15^{\prime}$ \\
\hline $\begin{array}{l}\text { Actividad de } \\
\text { contraste }\end{array}$ & $\begin{array}{l}\text { Cuestionario en Google forms. Repaso del } \\
\text { contenido de la sesión. }\end{array}$ & $5^{\prime}$ \\
\hline $\begin{array}{l}\text { Actividad de } \\
\text { contraste }\end{array}$ & $\begin{array}{l}\text { Análisis guiado de un plano de replanteo de } \\
\text { cubiertas planas. }\end{array}$ & $15^{\prime}$ \\
\hline $\begin{array}{l}\text { Actividad de } \\
\text { contraste }\end{array}$ & $\begin{array}{l}\text { Actividad en grupo de diseño del plano de } \\
\text { cubiertas de modelo de grupo. Corrección en } \\
\text { clase. }\end{array}$ & $25^{\prime}$ \\
\hline Práctica & $\begin{array}{l}\text { Para practicar en casa, se explica la actividad } \\
\text { individual sobre replanteo de cubierta plana para } \\
\text { la que tendrán una semana. }\end{array}$ & $5^{\prime}$ \\
\hline
\end{tabular}

Tabla 3. Secuencia de actividades de la tercera sesión de aplicación del CIMA.

\begin{tabular}{|l|l|c|}
\hline \multicolumn{2}{|c|}{ SESIÓN 3 $\left(120^{\prime}\right)$} \\
\hline ACTIVIDAD & DESCRIPCIÓN & TIEMPO \\
\hline $\begin{array}{l}\text { Actividad de } \\
\text { contraste }\end{array}$ & $\begin{array}{l}\text { Exposición de cada grupo de la tarea que } \\
\text { se realizó en clase la sesión anterior y no } \\
\text { dio tiempo a terminar. }\end{array}$ & $30^{\prime}$ \\
\hline
\end{tabular}

Ciclos de Mejora en el Aula (2020). Experiencias de Innovación Docente de la US Esta obra se distribuye con la licencia Creative Commons 


\begin{tabular}{|l|l|c|}
\hline Teoría & $\begin{array}{l}\text { Los contenidos básicos de la teoría de } \\
\text { cubiertas inclinadas se darán de forma } \\
\text { expositiva, intercalados continuamente } \\
\text { con preguntas para que los estudiantes } \\
\text { participen. Se usarán herramientas } \\
\text { digitales para realizar nubes de palabras, } \\
\text { cuestionarios parciales etc. }\end{array}$ & 20' \\
\hline $\begin{array}{l}\text { Actividad de } \\
\text { contraste }\end{array}$ & $\begin{array}{l}\text { Análisis guiado de un ejemplo de replanteo } \\
\text { de cubierta inclinada. }\end{array}$ & 20' \\
\hline $\begin{array}{l}\text { Actividad de } \\
\text { contraste }\end{array}$ & $\begin{array}{l}\text { Actividad en grupo de replanteo de una } \\
\text { cubierta inclinada. Presentación al final } \\
\text { de la clase. }\end{array}$ & 20' trabajo + \\
\hline 15' exposición \\
\hline $\begin{array}{l}\text { Actividad de } \\
\text { contraste }\end{array}$ & $\begin{array}{l}\text { Para practicar en casa, se explica la } \\
\text { actividad individual sobre replanteo de } \\
\text { cubierta inclinada para la que tendrán una } \\
\text { semana. }\end{array}$ & 5' \\
\hline
\end{tabular}

Tabla 4. Secuencia de actividades de la cuarta sesión de aplicación del CIMA.

\begin{tabular}{|c|c|c|}
\hline \multicolumn{3}{|c|}{ SESIÓN 4 (120') } \\
\hline ACTIVIDAD & DESCRIPCIÓN & TIEMPO \\
\hline $\begin{array}{l}\text { Actividad de } \\
\text { contraste }\end{array}$ & $\begin{array}{l}\text { Repaso en pizarra de la actividad realizada } \\
\text { en casa. Resolución de dudas de la sesión } \\
\text { anterior. }\end{array}$ & $40^{\prime}$ \\
\hline Teoría & $\begin{array}{l}\text { Se aporta la información básica } \\
\text { (normativa principalmente) necesaria para } \\
\text { esta sesión, centrada en el contenido de } \\
\text { resolución de detalles constructivos de } \\
\text { puntos singulares. }\end{array}$ & $20^{\prime}$ \\
\hline $\begin{array}{l}\text { Actividad de } \\
\text { contraste }\end{array}$ & $\begin{array}{l}\text { En grupos, se plantea una actividad de } \\
\text { resolución de puntos singulares de un } \\
\text { modelo de edificio. Lo realizan en clase } \\
\text { y se corrige en pizarra. Cada grupo tiene } \\
\text { que resolver detalles de huecos, cubierta } \\
\text { inclinada y cubierta planta. }\end{array}$ & $40^{\prime}$ \\
\hline $\begin{array}{l}\text { Cuestionario } \\
\text { final }\end{array}$ & $\begin{array}{l}\text { Los alumnos realizarán nuevamente } \\
\text { el cuestionario que se les hizo al } \\
\text { principio para analizar la evolución de su } \\
\text { aprendizaje. }\end{array}$ & $20^{\prime}$ \\
\hline
\end{tabular}

Ciclos de Mejora en el Aula (2020). Experiencias de Innovación Docente de la US Esta obra se distribuye con la licencia Creative Commons 


\section{Cuestionarios inicial y final.}

El cuestionario se ha proporcionado a los alumnos por medio de la aplicación Google Forms, debido a la imposibilidad de pasarlo en papel procedente de las medidas de la escuela ante el estado de pandemia por el COVID-19. Los estudiantes han respondido las mismas preguntas al comienzo de la primera clase y al final de la última clase del CIMA, para poder evaluar el progreso de su aprendizaje.

Las preguntas se han planteado a partir de las preguntas que estructuraban el mapa de contenidos (pregunta 1,2 y 4) más otra que trata de establecer los valores previos que ya tiene los alumnos con relación al concepto de sostenibilidad. De esta manera, las preguntas son:

1. ¿Qué elementos integran la envolvente o piel de un edificio?

2. ¿Para qué construimos los muros del edificio? ¿y las cubiertas?

3. Ordena de más a menos sostenible cada uno de los siguientes materiales y explica el porqué de tu decisión:

4. Cal, Yeso, Cemento, Poliuretano, Cerámica, Madera.

5. En el siguiente dibujo, indica qué se está representando, el nombre de cada uno de los elementos señalados (A y B) y su función dentro del conjunto.

Ciclos de Mejora en el Aula (2020). Experiencias de Innovación Docente de la US Esta obra se distribuye con la licencia Creative Commons 


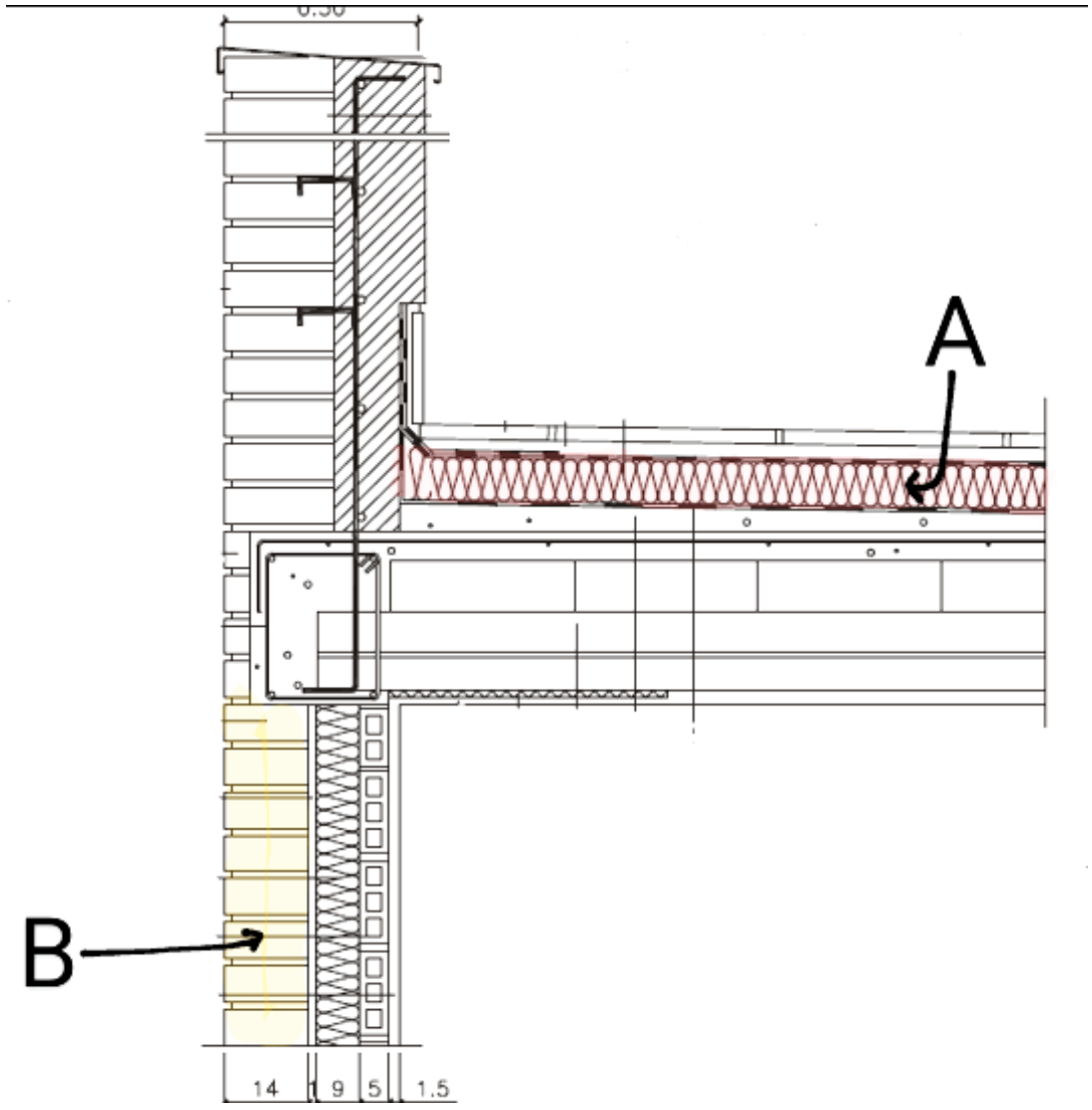

Figura 4. Detalle constructivo para la cuarta pregunta del cuestionario inicial y final.

\section{Aplicación del CIMA}

Para poder valorar de forma consciente y explícita el desarrollo de las sesiones, se hace uso del diario de clase, una herramienta profesional sencilla que permite tomar decisiones de manera fundamentada (Porlán, 2008). A continuación, se presenta el diario llevado durante las cuatro sesiones de aplicación del CIMA:

Diario de la 1ạ sesión (19/10/20): La primera sesión se inicia brevemente indicando a los alumnos que se empieza

Ciclos de Mejora en el Aula (2020). Experiencias de Innovación Docente de la US Esta obra se distribuye con la licencia Creative Commons 
un bloque nuevo en la asignatura bajo la pregunta “¿Cuáles son los diferentes sistemas del edificio?" y que se aplicará un diseño de la docencia innovador como parte de una actividad de mejora de la docencia universitaria. Para ello, se les presenta el cuestionario inicial que hacen online, pues la situación de pandemia no nos permite utilizar papel. Considero que el hecho de usar una plataforma que les es familiar (Google forms) y este formato online le quita peso al asunto de que es una evaluación, lo consideran más un juego. No se ve la típica cara de preocupación cuando parece que se les está examinando. A continuación, se procede a comenzar a presentar la primera pregunta “Pará qué se construye la envolvente del edificio?", a la cual se contesta con una actividad de contraste, la realización entre todos de una nube de palabras en la aplicación "mentimeter". El resultado es bueno, entre todos y mediante un par de preguntas por mi parte se contesta bastante bien a la pregunta. La participación es alta. Sigue la dinámica de juego en la clase.

A continuación, se pasa a la parte más árida, de teoría, que se plantea de forma que los alumnos vayan contestando a preguntas, aunque se nota que cuando las preguntas se lanzan al gran grupo nunca hay contestación directa, no sé si por timidez o por desconocimiento, pero las preguntas no son complejas. Al final me da la sensación de que la parte teórica se ha desarrollado más o menos a la manera tradicional, si bien se ha reducido a lo mínimo, durando unos 40 minutos. Por último, se trabajan las actividades de contraste.

La sensación general en la sesión es buena, si bien los tiempos inicialmente planteados no se han podido cumplir y me he sentido toda la sesión "corriendo", apresurándome en todas las actividades intentando que den tiempo, descuidando otros aspectos de la docencia. Por inercia, el tiempo dedicado a la explicación teórica ha sido más largo de lo previsto, si bien esto se ha visto afectado por querer que los alumnos participen y ellos no estar tan dispuestos.

Ciclos de Mejora en el Aula (2020). Experiencias de Innovación Docente de la US Esta obra se distribuye con la licencia Creative Commons 
Cuando se pregunta en clase en general la participación es baja. Sin embargo, la actividad que se realiza online y la actividad por grupos parece entusiasmarles. El análisis guiado en gran grupo no parece la mejor opción, o al menos estaría interesante darle más tiempo para realizarlo, pues es la parte en la que más pueden aprender. En general parece que están más dispuestos a participar si es de forma anónima (cuestionarios online, juegos, grupos pequeños) que si se les pide directamente que respondan mostrándose al grupo.

Diario de la 2â sesión (23/10/20): La dinámica planteada en clase es similar a la primera, introduciendo alguna mejora: para darle mayor interés a la necesaria parte teórica, se incluye un breve cuestionario final en Google forms para motivarles a que presten atención y pregunten dudas. Por otro lado, visto que cada vez hay más alumnos que tienen la necesidad de seguir la clase online, y ante la gran cantidad de actividades que se plantean en grupo, se van a crear subgrupos en enseñanza virtual para que tengan un Collaborate privado para poder trabajar. Para enseñarles esta dinámica, se plantea una actividad corta y muy sencilla con contenido del bloque para que utilicen la herramienta. El resto de la estructura de la clase es similar a la anterior.

Esta vez la clase se ha llevado con mayor soltura, con predominancia del trabajo por parte de los estudiantes. Sin embargo, el número de actividades planteadas ha sido excesivo. No ha dado tiempo a corregir en clase. Además, ciertos problemas técnicos por parte tanto mía como de los alumnos ha prolongado mucho el tiempo dedicado a la actividad que se pretendía corta de manejo de la herramienta. Decido que es mejor menos actividades y más tiempo para cada una, cosa que modifica la secuencia de actividades de la siguiente sesión. Sin embargo, estoy muy contenta con la respuesta de los estudiantes a las actividades en grupo. También estoy contenta con el uso

Ciclos de Mejora en el Aula (2020). Experiencias de Innovación Docente de la US Esta obra se distribuye con la licencia Creative Commons 
de Blackboard. Mediante la aplicación han podido trabajar en grupos incluso los que están en casa. La actividad del cuestionario de Google para el repaso de contenidos es muy interesante porque mantiene la atención de los estudiantes durante la parte que podría ser más tediosa para ellos porque saben que se les va a evaluar inmediatamente. Considero que tengo que mejorar el material que les aporto a los alumnos si se reduce la clase teórica y se van a basar más en ellos mismos de aquí en adelante. Me sugieren la idea de guiones de trabajo que pretendo implementar.

Diario de la 3á sesión (26/10/20): La clase comienza con la presentación y corrección en grupo de la actividad enviada la sesión anterior. Esta actividad es muy interesante pues los alumnos ven los errores de los compañeros y los suyos propios, y preguntan muchas dudas. Después de la parte teórica, de nuevo terminada con un pequeño cuestionario, la actividad de análisis de plano se plantea con un pequeño guion que se les aporta a los estudiantes, siendo así más claro y ordenado. La actividad final en grupo de replanteo de cubierta inclinada ha sido un éxito, esta vez ha dado tiempo a que la terminen en clase y ver algunos ejemplos.

El ritmo de la clase ha sido esta vez mucho más tranquilo y los alumnos han podido desarrollar mejor los ejercicios en la clase. He sido más consciente de mí misma a la hora de explicarme, pues no estaba tan pendiente del tiempo para las actividades. Creo que las exposiciones del trabajo de los alumnos les ayudan mucho a todos a resolver sus dudas, que suelen ser comunes. A pesar del menor número de actividades la clase sigue siendo muy dinámica y considero que los alumnos lo agradecen, las dos horas de clase se pasan muy amenas y están continuamente trabajando.

Ciclos de Mejora en el Aula (2020). Experiencias de Innovación Docente de la US Esta obra se distribuye con la licencia Creative Commons 
Diario de la 4ạ Sesión (4/11/20): En esta última sesión se repasan muchas de las cuestiones de las sesiones anteriores, pues la parte nueva de contenido no es tan extensa y se prefiere reforzar lo más importante de sesiones anteriores. Se hace mediante una actividad de presentación al grupo de correcciones de las actividades que han realizado en casa, con los fallos más comunes. Después, al igual que en clases anteriores procedemos a la breve parte teórica y la actividad de contraste de realización en grupos de detalles de cubiertas. Por último, se realiza el cuestionario final del CIMA.

Después de cuatro sesiones en las que he aplicado una estructura similar con los cambios que he ido viendo necesarios, creo que la dinámica de clase es muy buena y ya me siento cómoda con ella. Veo que los alumnos también se han adaptado y cada vez se ven más cómodos y trabajan mejor en grupos. Estoy contenta por haber podido llevarla incluso con los alumnos online, que parecen mostrarse bastante agradecidos por la atención que se les presenta incluso estando en casa. Es muy agradable ver cómo se conecta gente en casa y en clase para realizar las actividades en grupo. La sensación final es de éxito, siempre mejorable, pero al fin y al cabo ha sido posible realizar lo planteado.

\section{Evaluación del aprendizaje de los estudiantes}

Para la evaluación del aprendizaje de los alumnos, se analizan las respuestas a cada pregunta del cuestionario y se establecen niveles de aprendizaje en función de sus ideas previas y finales. En la tabla 5 se definen los niveles detectados en cada pregunta y los obstáculos que se detectan para pasar de uno a otro:

Ciclos de Mejora en el Aula (2020). Experiencias de Innovación Docente de la US Esta obra se distribuye con la licencia Creative Commons 


\begin{tabular}{|c|c|c|c|c|c|c|}
\hline & Nivel 1 & \multicolumn{2}{|l|}{ Nivel 2} & \multicolumn{2}{|l|}{ Nivel 3} & Nivel 4 \\
\hline \multirow[t]{2}{*}{ 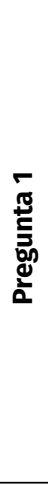 } & $\begin{array}{l}\text { Responde a la } \\
\text { pregunta sin } \\
\text { razonar qué es } \\
\text { la envolvente, } \\
\text { citando } \\
\text { elementos } \\
\text { aleatorios del } \\
\text { edificio. }\end{array}$ & \multicolumn{2}{|c|}{$\begin{array}{l}\text { Identifica parcialmente } \\
\text { los elementos de la } \\
\text { envolvente del edificio. }\end{array}$} & \multicolumn{2}{|c|}{$\begin{array}{l}\text { Identifica correctamente } \\
\text { los elementos, pero sin } \\
\text { nombrarlos con una } \\
\text { nomenclatura técnica } \\
\text { adecuada. }\end{array}$} & $\begin{array}{l}\text { Identifica los } \\
\text { elementos y } \\
\text { es capaz de } \\
\text { nombrarlos } \\
\text { adecuadamente. }\end{array}$ \\
\hline & \multicolumn{2}{|c|}{$\begin{array}{l}\text { Obstáculo: Entender el } \\
\text { concepto de envolvente o piel }\end{array}$} & \multicolumn{2}{|c|}{$\begin{array}{l}\text { Obstáculo: Conocer } \\
\text { los elementos } \\
\text { que componen la } \\
\text { envolvente }\end{array}$} & \multicolumn{2}{|c|}{$\begin{array}{l}\text { Obstáculo: Conocer el lenguaje } \\
\text { técnico apropiado }\end{array}$} \\
\hline \multirow{2}{*}{ 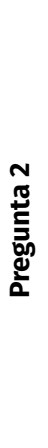 } & $\begin{array}{l}\text { No identifica } \\
\text { ninguna de las } \\
\text { funciones que } \\
\text { se le piden. }\end{array}$ & \multicolumn{2}{|c|}{$\begin{array}{l}\text { Identifica alguna } \\
\text { función principal de } \\
\text { muros y/o cubiertas. }\end{array}$} & \multicolumn{2}{|c|}{$\begin{array}{l}\text { Es capaz de entender la } \\
\text { multifuncionalidad de la } \\
\text { envolvente aportando varias } \\
\text { funciones importantes. }\end{array}$} & $\begin{array}{l}\text { Identifica } \\
\text { todas las } \\
\text { funciones más } \\
\text { importantes de } \\
\text { las envolventes } \\
\text { (firmeza, utilidad, } \\
\text { estética) }\end{array}$ \\
\hline & \multicolumn{2}{|c|}{$\begin{array}{l}\text { Obstáculo: Falta de capacidad } \\
\text { de racionamiento }\end{array}$} & \multicolumn{2}{|c|}{$\begin{array}{l}\text { Obstáculo: Entender } \\
\text { que las funciones } \\
\text { muros y cubiertas son } \\
\text { múltiples. }\end{array}$} & \multicolumn{2}{|c|}{$\begin{array}{l}\text { Obstáculo: Capacidad para } \\
\text { agrupar las múltiples funciones } \\
\text { en las tres principales } \\
\text { categorías. }\end{array}$} \\
\hline \multirow[t]{2}{*}{ 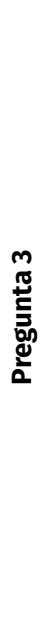 } & $\begin{array}{l}\text { No tiene } \\
\text { ningún criterio } \\
\text { de valoración } \\
\text { de la } \\
\text { sostenibilidad } \\
\text { de los } \\
\text { materiales. }\end{array}$ & \multicolumn{2}{|c|}{$\begin{array}{l}\text { Identifica un criterio de } \\
\text { sostenibilidad. }\end{array}$} & \multicolumn{2}{|c|}{$\begin{array}{l}\text { Es capaz de entender } \\
\text { que la sostenibilidad de } \\
\text { un producto depende de } \\
\text { varios factores, tanto de } \\
\text { fabricación como transporte, } \\
\text { durabilidad, capacidad de } \\
\text { reciclaje etc. }\end{array}$} & $\begin{array}{l}\text { Además de } \\
\text { entender la } \\
\text { complejidad } \\
\text { del concepto, } \\
\text { es capaz de } \\
\text { aplicarlo a } \\
\text { los materiales } \\
\text { aportados y } \\
\text { establecer un } \\
\text { orden coherente } \\
\text { y justificado. }\end{array}$ \\
\hline & \multicolumn{2}{|c|}{$\begin{array}{l}\text { Obstáculo: Entender el } \\
\text { concepto de sostenibilidad. }\end{array}$} & \multicolumn{2}{|c|}{$\begin{array}{l}\text { Obstáculo: Entender } \\
\text { la complejidad de la } \\
\text { sostenibilidad en sus } \\
\text { múltiples factores que } \\
\text { intervienen. }\end{array}$} & \multicolumn{2}{|c|}{$\begin{array}{l}\text { Obstáculo: Conocer el ciclo de } \\
\text { vida de los diferentes materiales }\end{array}$} \\
\hline
\end{tabular}

Ciclos de Mejora en el Aula (2020). Experiencias de Innovación Docente de la US Esta obra se distribuye con la licencia Creative Commons 


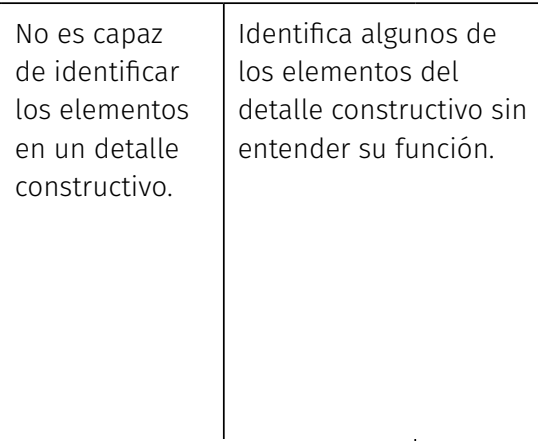

Obstáculo: Entender el concepto de sostenibilidad.

\section{Es capaz de identificar} elementos y función de los detalles constructivos.

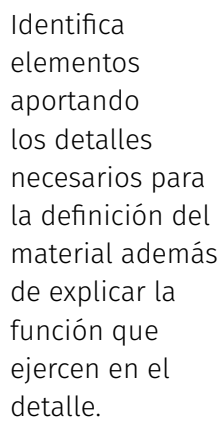

Obstáculo: Conocer el ciclo de vida de los diferentes materiales la complejidad de la sostenibilidad en sus múltiples factores que intervienen

Los resultados de los cuestionarios se muestran en la figura 5. En todas las preguntas se detecta una reducción en el porcentaje de alumnos en el nivel inferior y un aumento en los superiores, mostrando una evolución en los conocimientos generales del grupo. Sin embargo, la pregunta 2 y 3 no muestran un resultado muy satisfactorio, siendo pocos o ningún alumno los que alcanzan el nivel más alto. También en la pregunta 1, a pesar de que se han reducido, sigue existiendo un cuarto de la clase que no supera el nivel inferior.

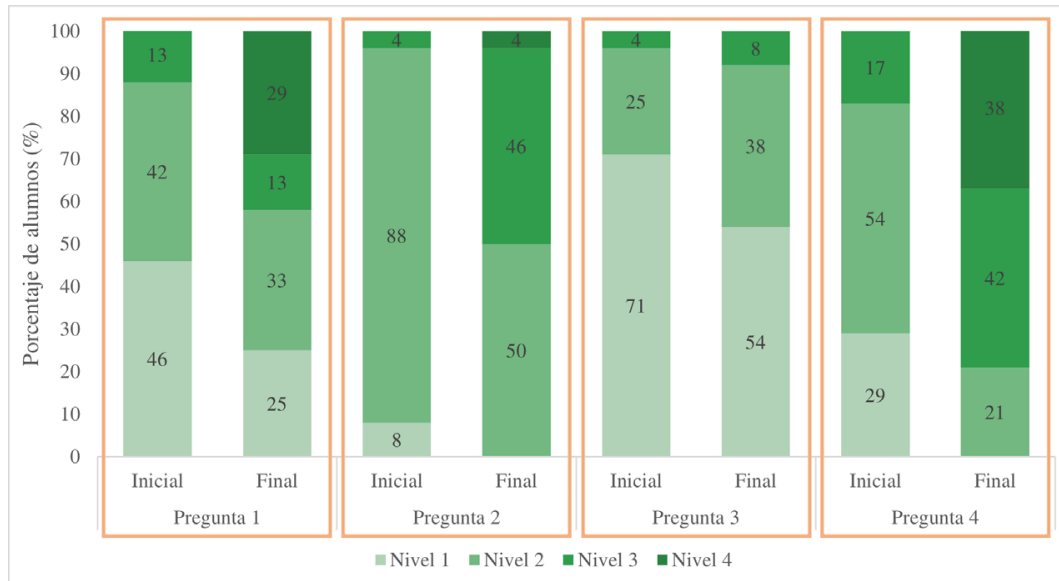

Figura 5. Resultados de las encuestas inicial y final en el grupo de alumnos.

Ciclos de Mejora en el Aula (2020). Experiencias de Innovación Docente de la US Esta obra se distribuye con la licencia Creative Commons 
Para mostrar el desarrollo individual de cada alumno, se han representado los sujetos en los diferentes niveles en cada uno de los cuestionarios en la tabla 6. En rojo se representan los estudiantes que no han sido capaces de avanzar de nivel en cada pregunta. Esta representación es muy interesante, pues indica a simple vista dónde se encuentran los escalones más difíciles de salvar en cada pregunta, que serán principalmente los que acumulen una mayor cantidad de individuos en rojo.

Tabla 6. Resultados individualizados de las encuestas inicial y final. En rojo, los individuos que no progresan en el aprendizaje en cada pregunta.

\begin{tabular}{|c|c|c|c|c|}
\hline & Nivel 1 & Nivel 2 & Nivel 3 & Nivel 4 \\
\hline P1 Inicial & $\begin{array}{l}1,2,4,11,12,13 \\
14,15,21,22,23\end{array}$ & $\begin{array}{c}5,6,8,9,10,16 \\
17,18,19,20\end{array}$ & $3,7,24$ & \\
\hline P1 Final & $1,11,12,13,15,22$ & $\begin{array}{c}2,4,8,9,10,14 \\
19,20\end{array}$ & $3,17,24$ & $5,6,7,16,18,21,23$ \\
\hline P2 Inicial & 1,11 & $\begin{array}{c}2,3,4,5,6,8,9 \\
10,12,13,14,15 \\
16,17,18,19,20 \\
21,22,23,24\end{array}$ & 7 & \\
\hline P2 Final & & $\begin{array}{c}2,4,8,10,14,15 \\
\begin{array}{c}16,17,19,20,21 \\
22\end{array}\end{array}$ & $\begin{array}{c}1,3,6,7,9,11,12 \\
13,18,23,24\end{array}$ & 5 \\
\hline P3 Inicial & $\begin{array}{c}1,2,3,4,5,8,9 \\
10,12,15,17,18 \\
19,20,21,22,23 \\
24\end{array}$ & $6,7,11,14,16,23$ & 13 & \\
\hline P3 Final & $\begin{array}{c}3,4,5,8,10,12 \\
15,17,18,19,20 \\
21,22\end{array}$ & $\begin{array}{c}1,2,6,7,11,14,16 \\
23,24\end{array}$ & 9,13 & \\
\hline P4 Inicial & $\begin{array}{c}2,6,8,10,17,22 \\
23\end{array}$ & $\begin{array}{c}1,3,4,5,7,9,13 \\
14,15,16,18,19 \\
21\end{array}$ & $11,12,20,24$ & \\
\hline P4 Final & & $2,3,10,15,21$ & $\begin{array}{c}1,4,5,8,12,13,14 \\
17,21,22\end{array}$ & $\begin{array}{c}6,7,9,11,16,18 \\
19,20,24\end{array}$ \\
\hline
\end{tabular}

Ciclos de Mejora en el Aula (2020). Experiencias de Innovación Docente de la US Esta obra se distribuye con la licencia Creative Commons 
En cuanto a las causas que han podido propiciar estos resultados, se puede decir que no se han trabajado lo suficiente la pregunta estructurante 2 (funciones de la envolvente) ni los conceptos actitudinales. Es evidente que a veces a los docentes nos parece que los obstáculos los van a encontrar en otros sitios, dejando de lado cuestiones más simples que luego muestran debilidades en los resultados. En cuanto a la pregunta 3, los problemas han sido dos: primero, se trata de un concepto actitudinal que se ha trabajado de manera transversal, sin actividad concreta para ello, con lo cual se ve que no es suficiente para que los estudiantes alcancen los objetivos. Por otro, el modo en que se ha redactado la pregunta hace que muchos alumnos la respondan parcialmente, de manera que no dan sus motivos para la clasificación realizada, siendo imposible analizar sus esquemas mentales.

En cuanto a la última pregunta, referente a los contenidos procedimentales, se considera un éxito ya que la mayor parte de los estudiantes se sitúan en los niveles superiores. Las actividades diseñadas para ellos han demostrado su efectividad.

\section{Evaluación del CIMA}

En relación a los contenidos, el tener un mapa desde el comienzo ha servido tanto al docente para estructurar las clases y las actividades de manera coherente como para los alumnos, como herramienta de ayuda para ir enlazando sus esquemas mentales. Un mapa de contenidos bien elaborado permite diseñar y relacionar las actividades con la evaluación y los contenidos de forma clara. El diseño de los mapas de contenidos es algo que considero esencial a mantener.

Ciclos de Mejora en el Aula (2020). Experiencias de Innovación Docente de la US Esta obra se distribuye con la licencia Creative Commons 
En relación a la metodología, a pesar de que algunas actividades diseñadas en sí mismas pueden mejorarse, la dinámica en clase ha funcionado muy bien, los alumnos han estado motivados la mayor parte del tiempo y las herramientas utilizadas, una vez superados los pequeños problemas técnicos, han sido esenciales para la parte del alumnado en cuarentena, demostrando que el trabajo síncrono es posible. Los cuestionarios tras la parte teórica mantienen una mayor atención y motivación en la parte más árida de la clase.

La secuencia de actividades planteada al principio era demasiado ambiciosa, los tiempos no estaban bien calculados pues no daba lugar a imprevistos técnicos u otras necesidades de los estudiantes. Se optó por dar más tiempo a las actividades y se tendrá en cuenta en CIMAs futuros.

Lo que se considera esencial es la presentación en clase por parte de los alumnos del trabajo realizado en aula. Ha sido la actividad más interesante para su aprendizaje y creo que se debe reservar tiempo para ello en todas las clases. La participación es alta, no como en la parte más teórica que ni aun preguntando a los alumnos directamente la participación es satisfactoria.

En relación a la evaluación, el uso del cuestionario inicial funciona muy bien para tener una idea de los conocimientos previos de los estudiantes y sirve para adaptar los contenidos en base a ello. Sin embargo, hay que destacar la importancia de diseñar cuidadosamente las preguntas para poder captar adecuadamente las ideas iniciales de los alumnos. La evaluación continua que se ha llevado gracias a la presentación de los trabajos en grupo en el aula también ha servido para que los estudiantes puedan autocorregirse y para tener una idea general de cuáles son las dificultades generales del grupo.

Ciclos de Mejora en el Aula (2020). Experiencias de Innovación Docente de la US Esta obra se distribuye con la licencia Creative Commons 
El uso de las herramientas online viene para quedarse. Han resultado ser útiles y muy amigables con el trabajo de los estudiantes, que se sienten más cómodos así incluso que antes y aumenta la participación. Pienso incorporar este tipo de actividades aun cuando la situación permita prescindir de ellas. También las considero una herramienta que mejora la comunicación con los estudiantes y se adapta a sus circunstancias.

El diseño de las sesiones partiendo del mapa de contenidos y las preguntas estructurantes es muy interesante y creo que es algo que utilizaré de aquí en adelante. También el uso de cuestionarios iniciales, y el análisis que se realiza con ellos, intentando sistematizar la dinámica.

El trabajo de actividades de contraste en grupos pequeños y presentar en clase es una actividad que ha resultado muy productiva, tanto para ellos aprender como yo conocer rápido sus carencias más generales.

\section{Conclusiones}

Los principios didácticos en los que se ha basado el diseño del CIMA partían de ciertas problemáticas previas que se suelen dar en la asignatura, y que se tratan de solventar:

- Metodología: el modelo metodológico habitual es básicamente transmisivo, el alumno recibe un conocimiento de forma expositiva y lo aplica a unas prácticas. En la clase, su participación es nula y el profesor no tiene "feedback" hasta que entrega la práctica. El CIMA propuesto hace que los alumnos sean más participativos en clase y construyan su propio conocimiento a partir de una serie de actividades de ideas previas y de contraste alternadas con teoría, que además han servido al profesor de continua información sobre los conocimientos de sus estudiantes.

Ciclos de Mejora en el Aula (2020). Experiencias de Innovación Docente de la US Esta obra se distribuye con la licencia Creative Commons 
- Evaluación: El diseño de las actividades de contraste que los alumnos deben realizar en clase aporta información al profesor que los utilizará como evaluación continua, no restringiéndose a las prácticas individuales y al examen final. De este modo se busca una motivación continua para los estudiantes y una mayor información para poder adaptar las clases al proceso de aprendizaje necesario.

- Trabajo en grupo y aprendizaje colectivo: Se ha incrementado el número de actividades que los alumnos realizan en grupos de hasta 6 personas, para que también se apoyen entre ellos en el aprendizaje y ganen otras competencias que solo el trabajo en grupo proporciona.

- Contenidos: Se ha introducido explícitamente la educación en valores: los contenidos actitudinales suelen ser ignorados especialmente en los primeros cursos. Se ha tratado de dar mayor predominancia, si bien este es un aspecto que se ha demostrado necesita mejorar.

- Herramientas digitales: debido a la situación de pandemia y la situación de aislamiento en la que se encuentra una parte de la clase, tanto las explicaciones como las actividades en clase se han realizado de manera sincrona. Esto supone una adaptación de toda la metodología a las herramientas digitales disponibles y se ha demostrado posible e incluso beneficioso en algunas cuestiones.

Aparte de todo ello, y de cara al futuro, se ha considerado el uso de la clase invertida una herramienta que puede tener gran beneficio sobre todo en esta situación de clases sincronas y en este curso, primero, donde los alumnos llegan con casi ningún conocimiento previo y este tipo de metodología ampliaría las posibilidades de las actividades de contraste en clase. Se plantea como una posible cuestión a incorporar en futuros CIMAs, aparte de las mejoras anteriormente comentadas. 
Palabras clave: Construcción I; Grado en Arquitectura; docencia universitaria; experimentación docente universitaria; herramientas digitales.

Keywords: Building Construction I; Degree in Architecture Fundamentals; University teaching; University teaching experimentation; digital tools.

\section{Referencias bibliográficas.}

Bain, K. (2007). Lo que hacen los mejores profesores universitarios. Valencia: Publicaciones de la Universitat de Valencia.

Finkel, D. (2008). Dar clase con la boca cerrada. Valencia: Publicaciones de la Universitat de Valencia.

Blandón González, B. (2019). Propuesta del nuevo modelo didáctico aplicado en construcción. Jornadas De Formación E Innovación Docente Del Profesorado, (1), 5879. https://doi.org/10.12795/jidu.2018.i01.03

Ministerio de fomento (2019). CTE. Código técnico de la Edificación. Documento Básico HS. Salubridad. (n.d.). Retrieved November 16, 2020, from https://www.codigotecnico.org/pdf/Documentos/HS/DBHS.pdf

Porlán, R. (Coord.) (2017). Enseñanza Universitaria. Cómo mejorarla. Madrid: Morata.

Porlán, R. (2008). El diario de clase y el análisis de la práctica. Andalucía: Averroes. Red Telemática Educativa de Andalucía.

Ciclos de Mejora en el Aula (2020). Experiencias de Innovación Docente de la US Esta obra se distribuye con la licencia Creative Commons 